\title{
Fluid flow in fields of resistance
}

\section{J.R. Blake}

\begin{abstract}
The velocity profiles in volumes of either passive resistance or active transport are calculated for both $l$ inear and non-linear resistive models. These include fluid flows containing particles in a channel or pipe subject to either a steady or oscillatory pressure gradient, cilia induced transport, and laminar flow in a non-linear resistive shear layer. Resistive elements tend to substantially reduce the inertial phase lag component in oscillatory flows. Only small concentrations of particles are needed to reduce the flow field to the Darcy approximation. An 'active porous media' model for a cilia sublayer predicts accurate volecity profiles for dense concentrations of cilia (for example, on Opalina and in the lung).
\end{abstract}

\section{Introduction}

Fractical problems in widely differing fields of research ranging from soil physics, environmental mechanics to biological fluid mechanics have the common feature that we are considering fluid flow in fields of either passive resistance or active transport: for example, resistive flow in porous media, air motion within crop canopies, active transport by cilia in mucous movement and possible long distance sucrose translocation by micro-filaments in phloem cells. We could further extend the problem to suspensions in which the particulate matter is moving with the fluid. In this case for neutrally bouyant particles the force and couple on the particle will be zero (the particle will move with the local velocity and rotate at half the local vorticity of the fluid), but the straining motion due to rotation and higher moments will affect the ambient flow field.

Received 23 May 1975. 
It is extremely difficult to calculate the exact flow pattern around all the particles of the suspension, fixed or otherwise. To overcome this serious drawback we may investigate the problem in terms of ensemble averages, which are denoted by angle brackets. Thus mathematically the problem is defined by an ensemble average of the Navier Stokes equations, with a force term which is a functional of the velocity. That is, for an incompressible fluid, the equations of motion are

$$
\begin{aligned}
\rho\left\langle\frac{\partial u}{\partial t}+u . \nabla u\right\rangle & =-\langle\nabla p\rangle+\mu\left\langle\nabla^{2} u\right\rangle+\langle F[u]\rangle, \\
\langle\nabla . u\rangle & =0,
\end{aligned}
$$

where $u$ is the velocity vector, $p$ the pressure, and $\rho$ and $\mu$ the density and viscosity respectively. The force term $\langle F[u]\rangle$ represents the resistive or active element of the flow field. The problems considered in this paper will be either uni-directional flows or flows at low Reynolds numbers such that the advective terms in (1) will be equated to zero. However $F[u]$ may still be non-linear, especially for larger Reynolds numbers, so the equations still maintain a non-linear character.

There have been some detailed analyses of (I) with respect to porous media (Saffman [11]). He defines

$$
U(x)=(u(x)) \text { and } P(x)=\langle p(x)) .
$$

The velocity $U$ is usually referred to in terms of a flux per unit area. The mean interstitial velocity $\bar{U}(x)$ and the mean interstitial pressure $\vec{p}(x)$ are conditional averages taken over the fluid alone and are given by (Saffman $[11])$ :

$$
\begin{aligned}
& \bar{U}(x)=\frac{1}{n}\langle H(x) u(x)\rangle=\frac{U(x)}{n}, \\
& \bar{p}(x)=\frac{1}{n}\langle H(x) p(x)\rangle=\frac{P(x)}{n},
\end{aligned}
$$

where $\eta=\langle H(x)\rangle$ with $H(x)$ defined as equal to one in the pores and zero in the solid. The ensemble averages $U(x)$ and $P$ are continuous and differentisble.

Furthermore, if we considered a function $\phi(x)$ which is piecewise smooth and has single jumps at discontinuities, then 
(4a)

$$
\left\langle\frac{\partial \phi}{\partial x_{i}}\right\rangle=\frac{\partial \phi\rangle}{\partial x_{i}}-\left\langle[\phi]_{i}\right\rangle
$$

where

$$
\left\langle[\phi]_{i}\right\rangle=\lim _{V \rightarrow 0}\left\langle\frac{1}{V} \int_{S} \phi n_{i} d S\right\rangle
$$

and

$$
\left\langle\frac{\partial \phi}{\partial t}\right\rangle=\frac{\partial\langle\phi\rangle}{\partial t}
$$

The velocity $U(x)$ is continuous across the surface $S$, so it immediately follows that

$$
\nabla \cdot U=0
$$

and $\left\langle\frac{\partial U_{i}}{\partial x_{j}}\right\rangle=\frac{\left.\partial U_{i}\right\rangle}{\partial x_{j}}$, since it is also continuous; then (I) becomes, for uni-directional flow,

$$
\rho \frac{\partial U_{i}}{\partial t}=-\frac{\partial P}{\partial x_{i}}+\mu \nabla^{2} U_{i}+F_{i}
$$

Saffman [11] derives this formulation by considering an earlier step: the divergence of the stress tensor to obtain the force. Because of the continuous nature of the functions it is in order for us to proceed directly from (I).

The complete definition of the problem is obtained if we define a further relation between $F$ and $U$. For low Reynolds number flows the equations are linear so we can express $F$ as a linear functional of $U$ as follows :

$$
F_{i}(\mathrm{x})=\mu \int_{t} \int_{V} K_{i j}\left(\mathrm{x}, \xi, t, t^{\prime}\right) U_{j}\left(\xi, t^{\prime}\right) d \xi d t^{\prime}-\bar{p} \nabla \eta
$$

This equation can be further simplified if we expand

$$
K_{i j}\left(x, \xi, t, t^{\prime}\right)=\left(K_{i j}(x) \delta(x-\xi)+K_{i j k}^{\prime} \delta^{(k)}(x-\xi)+\ldots\right) \delta\left(t-t^{\prime}\right)
$$

where $\delta^{(k)}(x-\xi)=\frac{\partial}{\partial x_{k}} \delta(x-\xi)$ and $\delta(x-\xi)$ is the Dirac delta function.

Equation (6) becomes, on substitution of (7) and (8), 


$$
\rho \frac{\partial U_{i}}{\partial t}=-\eta \frac{\partial \bar{p}}{\partial x_{i}}+\mu \nabla^{2} U_{i}-\mu K_{i j} U_{j}+\mu K_{i j k}^{\prime} \frac{\partial U_{j}}{\partial x_{k}} .
$$

This equation, (9), allows for not only resistive elements but also a term due to a couple or straining motion on the suspended particles. Higher order moments would correspond to higher order derivatives in (8). These are neglected in this paper because their influence on the flow-field is much smaller than the force and couple terms and al so from a practical point of view since these higher moments are difficult to measure. This equation is sufficient from the practical point of view since $\bar{p}$ and $U$ are the measurable quantities, although it clearly has statistical. limitations from the theoretical side. Equation (9) without the time dependent term and third order tensor $K_{i j k}$ has been used by Brinkman [3] in calculating the increased resistance on sedimenting spheres. If we neglect the laplacian term as well, (9) reduces to Darcy's equation for flow in porous media;

$$
K_{i j j}=-\frac{n}{\mu} \frac{\partial \bar{p}}{\partial x_{i}}
$$

In the next sections we will consider solutions to equations (9) for special cases which may have some practical relevance.

\section{Flow in a two-dimensional channel and a circular cylinder}

In this section we will consider problems of uni-directional flow in a two-dimensional channel or a circular cylinder. We consider flow fields in either a resistive regime (proportional to $U$ ) or a force free medium (proportional to $\partial U / \partial x$ ) for oscillatory pressure gradients. The steady flow solutions and resistance free solutions are special cases of the oscillatory pressure gradient resistive medium flow field.

(a) Resistive medium

A problem of practical interest is the flow profile in a twodimensional channel or circular cylinder in which there are particles resisting fluid motion. For example in filtration processes we need to consider the flow through arrays of cylinders. It also accours frequently in the biological community, for example, the translocation of sucrose in phloem cells of plants in which long fibres extend along the tube. These 
may assume either a passive or active role, as the exact nature of their function to the plant is not known.

Oscillatory flow is an important component of arterial blood flow because of the rhythmic beating of the heart. We can analyse the oscillatory component of this flow by considering an oscillatory pressure gradient $\frac{d \bar{p}}{d x}=K e^{i \omega t}$. Similar problems to this have been treated by Womersley [12], Jones [9], and Chow and Lai [4]. Thus the equations of motion with resistance for the two dimensional and axisymmetric cases are

$$
\begin{aligned}
& \eta K e^{i \omega t}=\mu \frac{\partial^{2} U}{\partial y^{2}}-\rho \frac{\partial U}{\partial t}-\gamma U, \\
& \eta K e^{i \omega t}=\mu\left(\frac{\partial^{2} U}{\partial r^{2}}+\frac{1}{r} \frac{\partial U}{\partial r}\right)-\rho \frac{\partial U}{\partial t}-\gamma U,
\end{aligned}
$$

respectively. The scalar $\gamma$ is the resistive parameter peculiar to the resistive medium and has the dimensions of force per unit volume. The solution of these equations subject to the no-slip condition on the walls is,

$$
U(y)=\frac{\eta}{\gamma} K e^{i \omega t}\left(\frac{\cosh \theta y^{\prime}}{\cosh \theta}-1\right),
$$

and

$$
U(r)=\frac{\eta}{\gamma} K e^{i \omega t}\left(\frac{I_{0}\left(\theta r^{\prime}\right)}{I_{0}(\theta)}-1\right),
$$

where

$$
\theta=\left(\frac{\gamma h^{2}}{\mu}+i \frac{\rho \omega h^{2}}{\mu}\right)^{\frac{1}{2}}=\left(\alpha^{2}+i \beta^{2}\right)^{\frac{1}{2}} ;
$$

where $\alpha=\left(\frac{\gamma h^{2}}{\mu}\right)^{\frac{1}{2}}$ and $\beta=\left(\frac{\rho h^{2}}{\mu}\right)^{\frac{2}{2}}$. We have defined $y^{\prime}=y / h$ in (12) and $r^{\prime}=r / h$ in (13) where $h$ is either channel height or pipe radius, and $I_{0}$ is the zeroth order modified Bessel function of the first kind. The parameter $B$ is commonly referred to as the womersley parameter or as $\beta^{2}$ the oscillatory Reynolds number. The special case of $\alpha$ and $\beta$ equal to zero together with $n=1$ reduces to the Poiseuille parabolic profile in both cases. 
Because of the similarity in velocity profiles for both the twodimensional and cylindrical models we will only illustrate the latter case. In Figure $I$ (a) we indicate the velocity profiles for various values of $(\alpha, \beta)$. The combination line (-.-) indicates the steady velocity profiles (that is, $\beta=0$ ) for various values of the resistance parameter $\alpha$. As $\alpha$ increases the velocity profile flattens out while the magnitude decreases. It can be seen for values of $\alpha$ greater than 8 that the velocity profiles become almost flat except near the walls where the noslip condition becomes important. For these larger values of $\alpha$ we can neglect the no-slip condition together with the higher derivatives in (II) and just consider the Darcy solution which is the line marked $D$ in Figure 1 (a). The oscillatory velocity profiles without resistance (that is, $\alpha=0$ ) are shown by the dashed lines in Figure 1 (a). Again, for increasing $B$ the magnitude of the velocity profile decreases and for larger values of $\beta$ the maximum amplitude of the flow field is not on the centre line for this instant in time $(t=0)$. This is due to inertial effects which produce a time-lag in the flow field relative to the applied pressure gradient; this being illustrated in Figure 1 (b). The combined effects of inertia and resistance produce interesting features in the flow field. For smaller values of $(\alpha, \beta)$, the effect of increasing either parameter is to reduce the magnitude of the velocity. For larger values of $\beta$ though, finite values of $\alpha$ have the effect of increasing the magnitude of the velocity on the centre line, this being produced by decreasing the phase lag which is clearly indicated by Figure 1 (b).

We can obtain an estimate of the particle concentration in the fluid medium for fixed particles by considering an array of cylindrical rods aligned with the flow for very small Reynolds numbers. In this case

$$
\gamma=\frac{2 \pi \mu}{\left(\log \frac{d}{r_{0}}-1.32\right) d^{2}},
$$

where $d$ is the spacing of the rods in the regular array and $r_{0}$ their radius (Happel and Brenner [7]). Thus the non-dimensional parameter $\alpha$ is equal to 


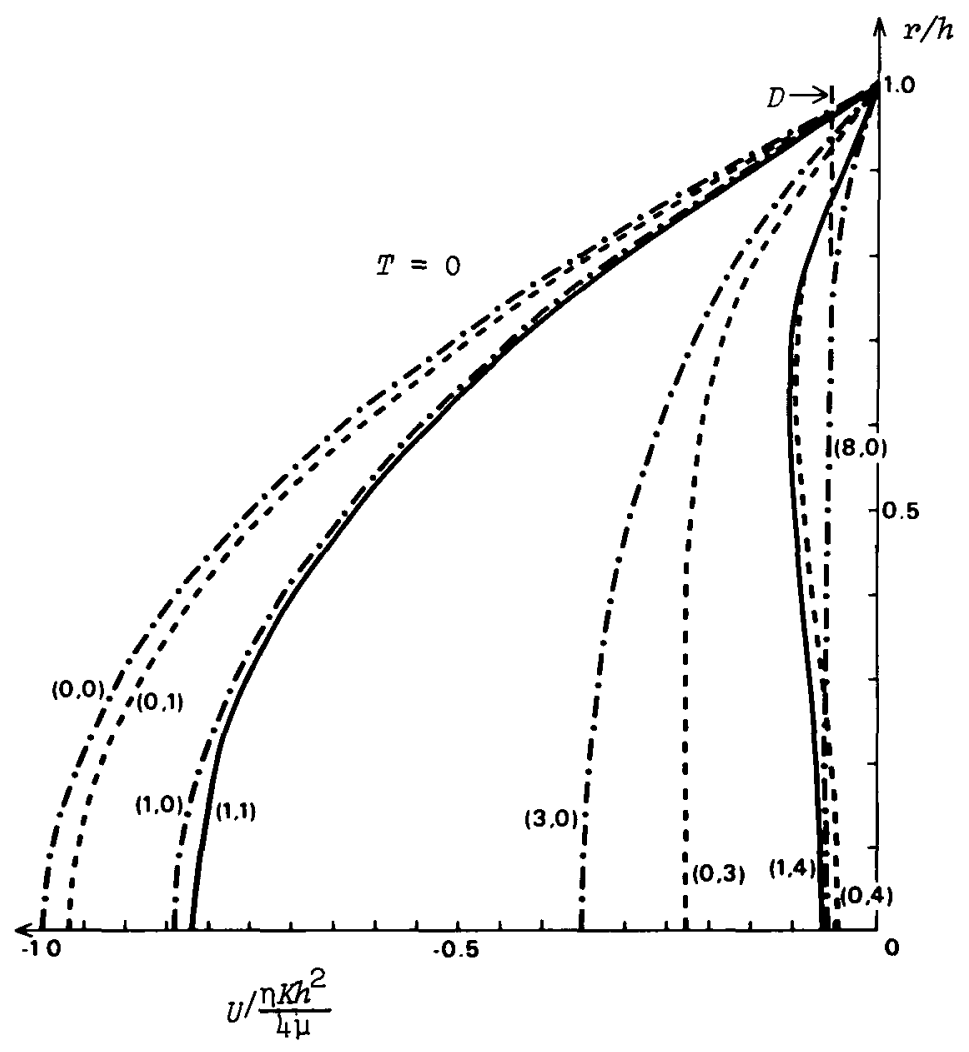

Figure 1 (a). Non-dimensionalised velocity profiles for different values of the parameters $(\alpha, \beta)$. Note the rapid decrease in the magnitude of the velocities for increasing values of $\alpha$ and B. 


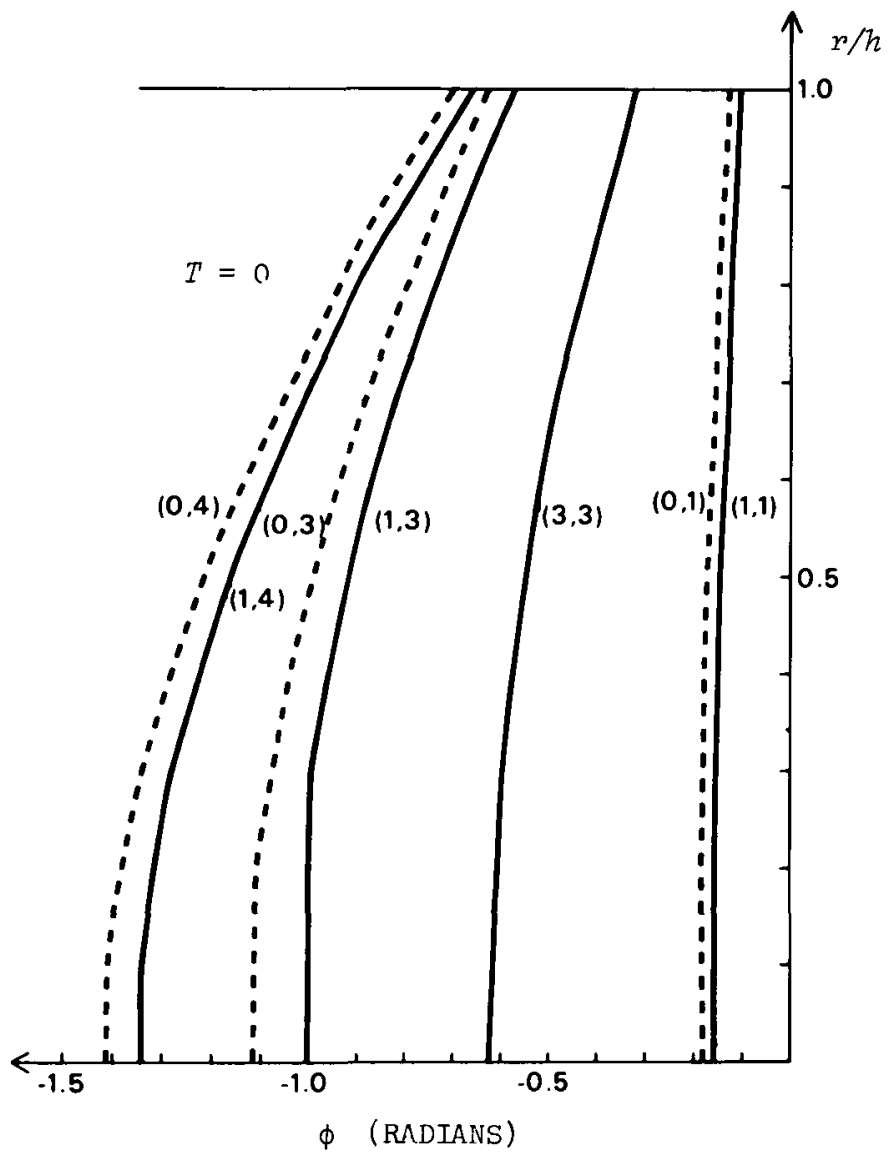

Figure 1 (b). Phase lag $\phi$ as a function of radius for different values of $(\alpha, \beta)$. The effect of increasing $\alpha$ is to decrease the phase lag $\phi$. 


$$
\alpha=\left|\frac{2 \pi h^{2}}{\left|\log \frac{d}{r_{0}}-1 \cdot 32\right| d^{2}}\right|^{\frac{3}{2}} .
$$

The volume concentration of rods is equal to

$$
c=\pi\left(\frac{x_{0}}{d}\right)^{2} \text {. }
$$

Thus if we took values of $r_{0} / d=0.1$ and $d / h=0.1$ we would produce values of $C=0.03$ and $\alpha=20$. From Figure 1 (a) it can be clearly seen that even at this low value volume concentration there are substantial changes in the flow field; in fact, even in this case, the Darcy model is more appropriate.

(b) Force-free medium

Many fluid flow problems involve the movement of suspensions of force free particles; for example blood flow, in which case the particles are being transported at the mean local fluid velocity and rotating with an angular velocity equal to half the local vorticity resulting in a dilatational motion and hence altering the velocity profile. By taking certain liberties with the model we can regard this as a rate-of-strain per unit volume. The equations of motion in this case for an oscillatory pressure gradient are

$$
n K e^{i \omega t}=\mu \frac{\partial^{2} U}{\partial y^{2}}+K^{*} \frac{\partial U}{\partial y}
$$

and

$$
n K e^{i \omega t}=\mu\left(\frac{\partial^{2} U}{\partial r^{2}}+\frac{1}{r} \frac{\partial U}{\partial r}\right)+K^{*} \frac{\partial U}{\partial r} .
$$

By using the definition of $\theta^{*}$ as being equal to

$$
\theta^{*}=\left(\frac{\alpha^{\prime 2}}{4}+i \beta^{2}\right)^{\frac{1}{2}}, \alpha^{\prime}=\frac{K^{*} h}{\mu}, \beta=\frac{\rho \omega h^{2}}{\mu},
$$

we can obtain the solution to (18) in the following form:

(21) $U(y)=\frac{i n K h^{2} e^{i \omega t}}{\mu \beta^{2}}\left(1-e^{-\alpha^{\prime} / 2}\left\{\frac{\cosh \alpha^{\prime} / 2}{\cosh \theta^{*}} \cosh \theta^{*} y^{\prime}+\frac{\sinh \alpha^{\prime} / 2}{\sinh \theta^{*}} \sinh \theta^{*} y^{\prime}\right\}\right)$, 
and the solution to (19) can be represented in terms of the following integral equation:

$$
U(r, t)=\frac{n h^{2} K e^{i \omega t}}{\mu} V\left(r^{\prime}\right),
$$

where $V\left(r^{\prime}\right)$ is the solution of

$$
V\left(r^{\prime}\right)=\frac{E_{1}\left(\alpha^{\prime}\right)-E_{1}\left(r^{\prime} \alpha^{\prime}\right)}{\alpha^{\prime 2}}-\frac{\left(1-r^{\prime}\right)}{\alpha^{\prime}}-\frac{1 n r^{\prime}}{\alpha^{\prime}}+i \beta^{2} \int_{0}^{1} K\left(r^{\prime}, \xi\right) V(\xi) d \xi,
$$

where $K\left(r^{\prime}, \xi\right)$ is defined as

$$
K\left(r^{\prime}, \xi\right)=\left\{\begin{array}{ll}
\xi e^{\alpha^{\prime} \xi}\left(E_{1}\left(\alpha^{\prime}\right)-E_{1}\left(\alpha^{\prime} \xi\right)\right), & 0 \leq r^{\prime}<\xi, \\
\xi e^{\alpha^{\prime} \xi}\left(E_{1}\left(\alpha^{\prime}\right)-E_{1}\left(\alpha^{\prime} r^{\prime}\right)\right), & \xi<r^{\prime} \leq 1 .
\end{array} .\right.
$$

The function $E_{1}(x)$ is an exponential integral defined to be equal to

$$
E_{1}(x)=\int_{x}^{\infty} \cdot \frac{e^{-t}}{t} d t
$$

Note $V\left(r^{\prime}\right)$ is complex, but the above integral can be resolved on the computer by using real arithmetic, if we define

$$
V\left(r^{\prime}\right)=V_{r}\left(r^{\prime}\right)+i V_{i}\left(r^{\prime}\right),
$$

and by resolving the problem into real and imaginary parts. Solutions are shown in Figure 2 for various values of $\left(\alpha^{\prime}, \beta\right)$. It can be clearly seen that the force-free terms above (that is, $B=0,(---)$ line in Figure 2) have a similar effect on the flow field as the resistive terms of the previous case. Again the phase-lag of the flow field due to the inertial terms is reduced by the action of the force-free terms; this being illustrated in Figure 2 by the continuous line (-).

\section{Applications to ciliary motion}

Cilia line the airways of the lung, our reproductive tubules, and propel many protozoa through their aqueous environment. They occur as a dense mat of elongated cylindrical tubules on the epithelial surfaces which beat with a "whiplash" movement. We can model the fluid motion in the cilia sublayer in terms of an "active porous medium" by neglecting the 


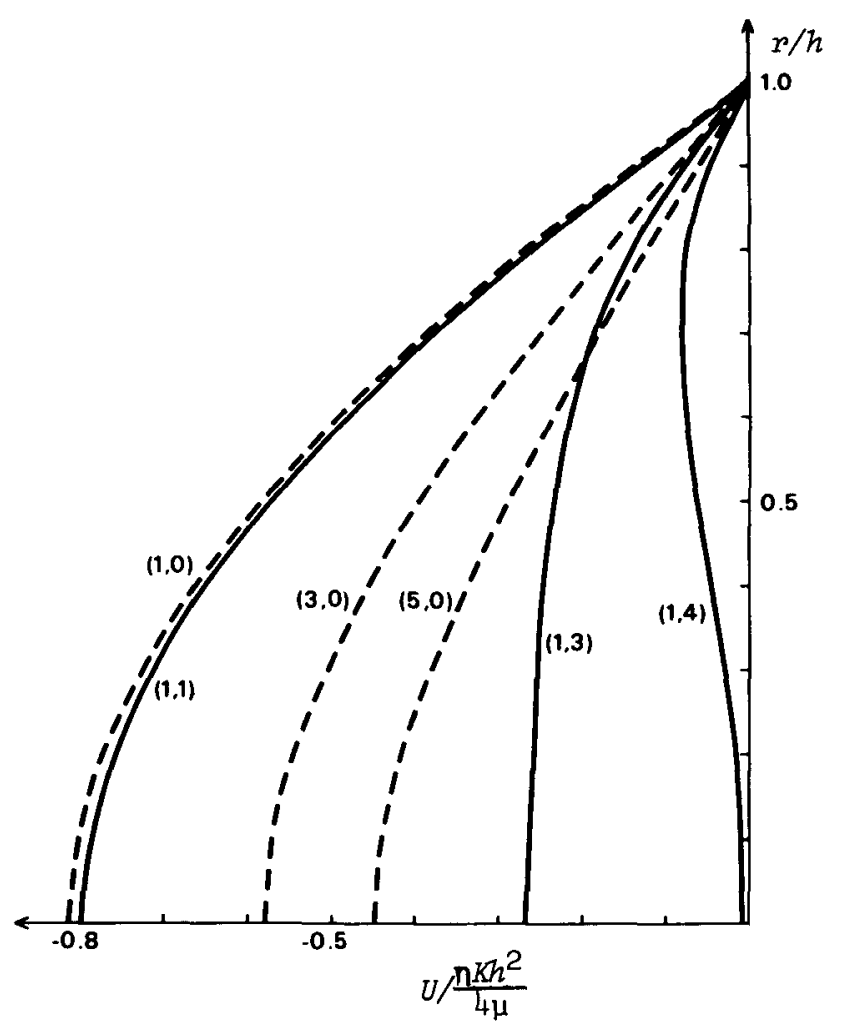

Figure 2. Non-dimensional velocity profiles for different values of $\left(\alpha^{\prime}, \beta\right)$. Similar to Figure 1, except that larger numerical values of $\alpha^{\prime}$ are required to decrease the magnitude of the velocity.

higher derivatives. Generally the fluid motion may be considered as quasisteady and without a pressure gradient. If we consider the porous media part of equations (6) and (7) by omitting time dependence, the problem reduces to solving

$$
\int_{V} K_{i j}\left(x, n^{\prime}\right)\left(U_{j}\left(\eta^{\prime}\right)-V_{j}^{*}\left(n^{\prime}\right)\right) d \eta^{\prime}=0,
$$

where $V_{j}^{*}\left(n^{\prime}\right)$ is the spatially averaged motion of the cilia and is defined 
as

$$
V_{j}^{*}=\frac{1}{1-\eta}\left\langle(1-H(x)) \frac{\partial \xi^{*}}{\partial t}\right\rangle=V_{j}\left(x_{3}\right),
$$

where $\xi^{*}$ is the vector cartesian position of the cilium at time $t$ for some arc length position $\boldsymbol{s}$. A similar equation to this was derived from the Stokes flow equations by Blake and sleigh [2]. The only meaningful coordinate dependence for cilia in (28) is where $V_{j}$ is a function of the vertical coordinate, in this case $x_{3}$.

We can obtain the function $V^{*}$ very easily numerically. Because of the periodicity of the cilia in space (metachronal wave) we need only average over time for one cilium to obtain the function $V^{*}$. From high speed movie films we can obtain the position of points equi-distant along a cilium at equal time intervals. Thus, if we consider $M$ points along the cilium and $N$ time intervals (see Figure $3(a)$ ) in a complete beat we can obtain the following simple sumation for the velocity profile for a twodimensional beat pattern:

$$
V_{k}\left(x_{3}^{j}\right)=\frac{L}{T} \sum_{n=1}^{N} \sum_{m=1}^{M} \frac{\xi_{k}^{*}\left(s_{m}, t_{n+1}\right)-\xi_{k}^{*}\left(s_{m}, t_{n}\right)}{W(j)},
$$

where in discretised terms

$$
x_{3}^{j}=\left[\frac{M}{2}\left(\xi_{3}^{*}\left(s_{m}, t_{n+1}\right)+\xi_{2}^{*}\left(s_{m}, t_{n}\right)\right)+0.5\right] L / M
$$

and $W(j)$ is the number of points in the interval $\left(j-\frac{1}{2}, j+\frac{1}{2}\right)$, the square brackets indicating integer part of the term inside. Thus $x_{3}^{j}$ is discretized into $M$ sections. $T$ is the period of the ciliary beat and $L$ the length of the cilium. For continuity reasons $V_{3}\left(x_{3}\right)=0$, since there is now flow through the solid boundary. If we make the gross assumption by using the first term only in (8), then

$$
U_{j}(x)=U_{j}\left(x_{3}\right) \text {. }
$$

That is the fluid is being propelled at the average velocity of the cilia. This assumption, like most porous media studies, neglects the local microscale fluid motion around the cilia, but produces a good macro-scale approximation as can be seen from Figure 3 (b) where the velocity profile 
for Opalina is shown. The observed velocity at the top of the cilia sublayer is usually about half the metachronal wave speed. In Opalina the frequency $f$ is $10-15 \mathrm{~Hz}$ and the wave length $\lambda$ is $12-15 \mu \mathrm{m}$, giving a wave speed of $120-225 \mathrm{um} / \mathrm{sec}$. This model predicts the velocity at the top layer as being equal to $0.5 \mathrm{fL}$, where $L$ is the length of the cilia, which in the case of opalina is $15 \mathrm{um}$. On substitution of these values the predicted theoretical velocity will be in the range $75-120 \mu \mathrm{m} / \mathrm{sec}$, being in good agreement with the observed values. To date, no observations have been made of the velocity profile within the sublayer of Opalina but we would qualitatively expect one similar to the predicted one; backflow being produced by the cilia in their recovery stroke.

This simple formula should be of great value to biologists and physiologists for calculating velocity profiles in dense mats of cilia instead of reverting to the complicated mathematical expressions derived previously (Blake [1]).

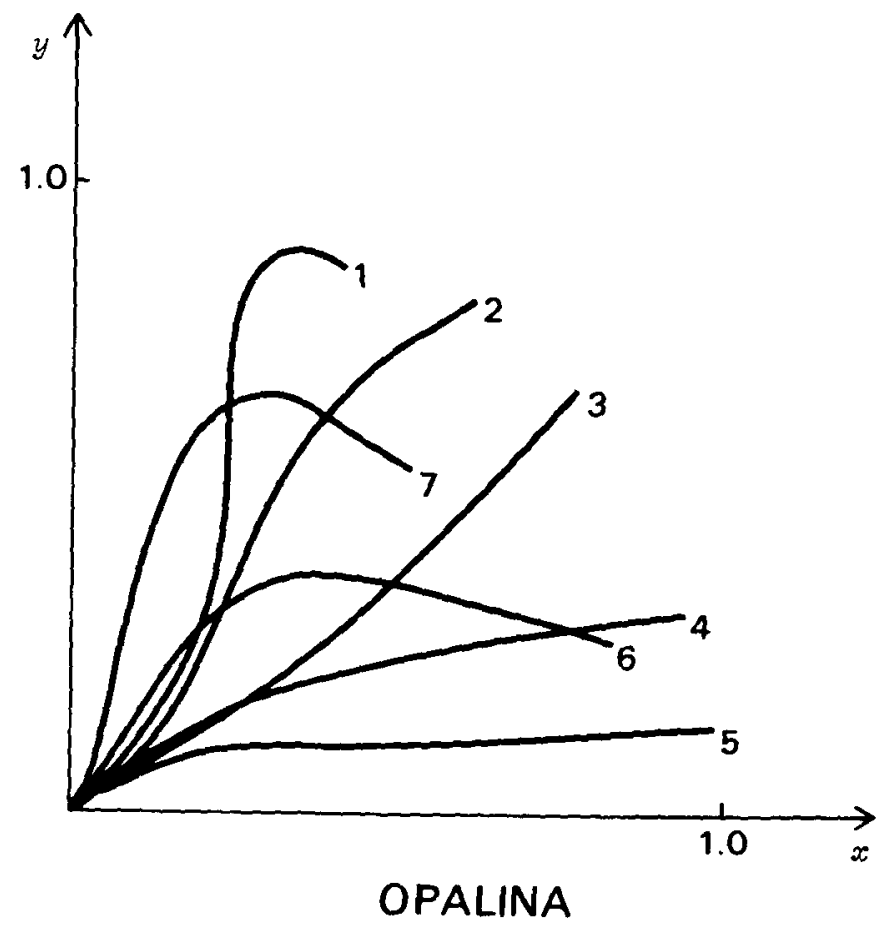

Figure 3 (a). Ciliary beating pattern for the protozoan Opalina. Numbering indicates successive equi-spaced time steps. 


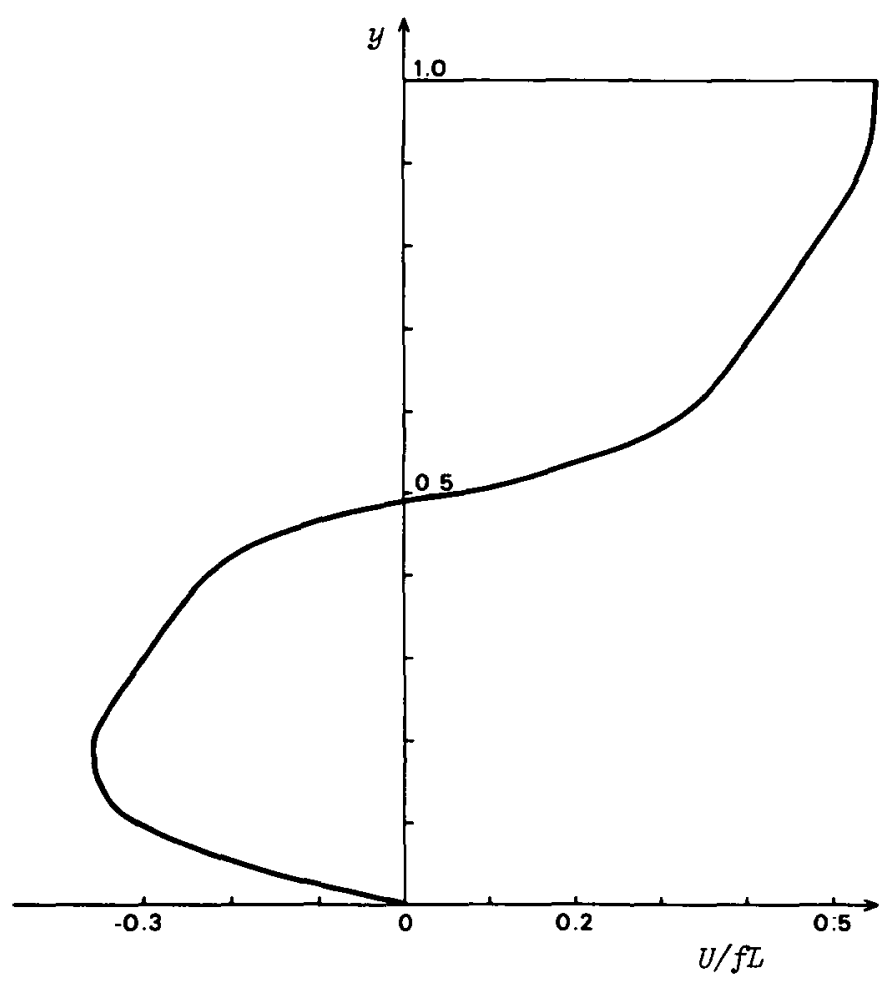

Figure 3 (b). Non-dimensionalised velocity profile using the 'active porous media' model.

\section{Laminar flow}

Although the flow-field in a crop canopy is nearly always turbulent, we can investigate the laminar flow problem in the hope that we may obtain some feeling for the physical problem. Let us suppose we have laminar unidirectional flow in a field of resistance without a mean pressure gradient: the driving mechanism being the flow field at the top of the crop canopy, in the form of a continuous velocity or a known shear stress. Thus the equations of motion and boundary conditions reduce to, in nondimensional form,

$$
\frac{d^{2} U}{d y^{2}}-\alpha * U^{2}=0, \quad U(0)=0 \text {, and } U(1)=1 \text {. }
$$

The solution of this equation can be obtained exactly in terms of 
weierstrassian elliptic functions $P(y ; a, b)$ (see, for example, Murphy! [10], p. 380, and Erdélyi et al [6], p. 328), but we prefer to approach the solution in terms of an asymptotic expansion of the form

$$
U(y)=\sum_{n=0} \alpha^{*} U_{n}(y)
$$

On substitution into (31) we obtain the following solutions

$$
\begin{aligned}
U_{0}(y) & =y, \\
U_{1}(y) & =y\left(y^{3}-1\right) / 12, \\
& \cdot \cdot \\
U_{n}(y) & =\sum_{k=0}^{n-1} \int_{0}^{1} K(x, \xi) U_{k}(\xi) U_{n-k-1}(\xi) d \xi,
\end{aligned}
$$

where

$$
K(x, \xi)= \begin{cases}x(\xi-1), & 0 \leq x<\xi, \\ \xi(x-1), & \xi<x \leq 1 .\end{cases}
$$

Convergence of the series is slow for large values of $\alpha^{*}$, and as many as 1000 terms are needed to provide convergence for $\alpha^{*}=5$.

Solutions for various values of $\alpha^{*}$ are shown in Figure 4. The shear is considerably reduced in the lower layers reaching a maximum at the canopy upper surface. If the flow field is turbulent, and the dynamic viscosity $\mu$ is replaced by an eddy viscosity an almost identical equation is obtained. For the turbulent case $\alpha^{*}$ would be in the range 4 to 6 for most cereal crops (for example, wheat, rice) and the velocity profile predicted by Cowan [5] is similar in shape to that in Figure 4. Further solutions to the turbulent problem have been considered in Inoue [8] and Cowan [5].

\section{Conclusions}

In this paper, analytic solutions to various physical problems involving flow in resistive media have been obtained. From the solutions for two-dimensional channel and cylindrical pipe flow through a resistive medium it was found that for even small volume concentrations the Darcy model for porous media was more appropriate. Resistive elements, as 


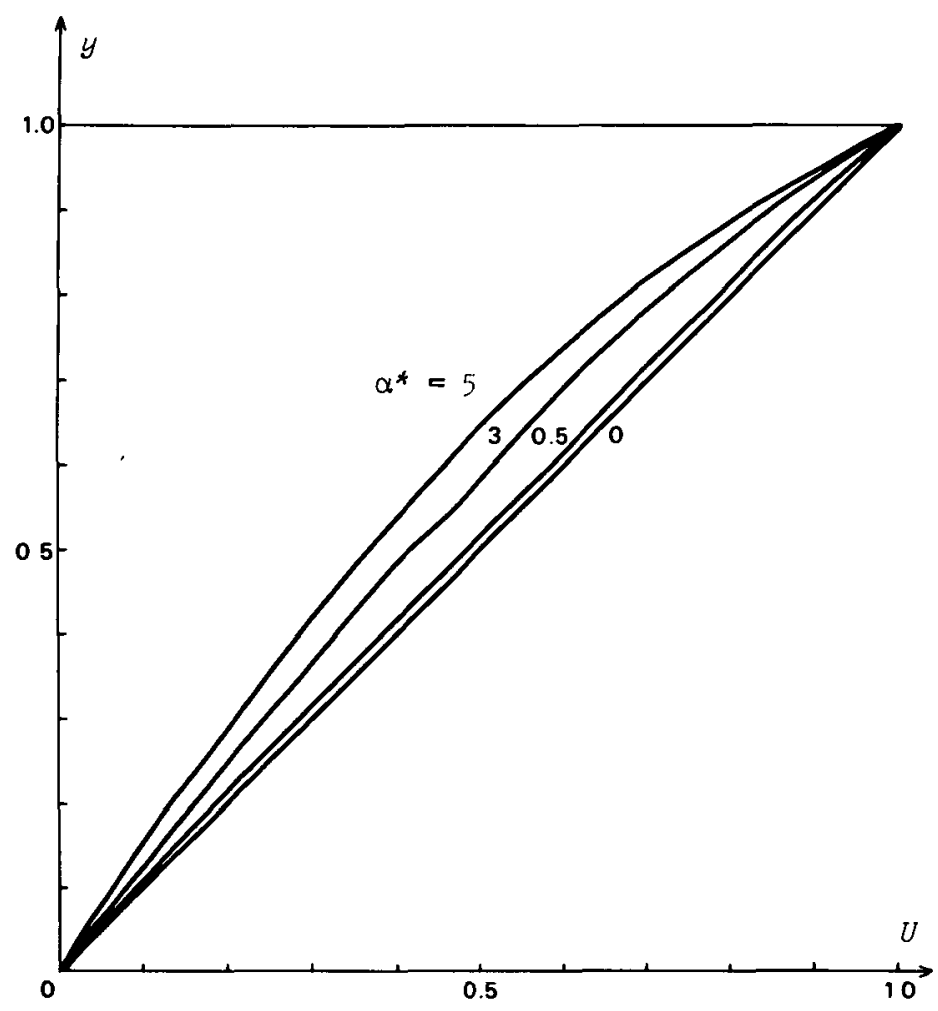

Figure 4. Velocity profiles in the non-linear laminar shear layer. Values of the non-dimensional parameter $\alpha^{*}$ are shown. $\alpha^{*}=0$ corresponds to constant shear (Couette flow).

expected, reduce the inertial phase lag for flows subjected to oscillatory pressure gradients.

A porous media model for flow due to cilia was found to be in agreement with previously proposed models for dense concentrations of cilia on the epithelia (for example, Opalina or in the lung). In the penultimate section laminar flow in a non-linear resistive medium was considered.

In conclusion this analysis shows that many important phenomena exist in flow-fields which include resistance to fluid motion. 


\section{References}

[1] John Blake, "A model for the micro-structure in ciliated organisms", J. Fluid Mech. 55 (1972), 1-23.

[2] J.R. Blake and M.A. Sleigh, "Hydro-mechanical aspects of ciliary propulsion", Proc. Symp. Mech. Swimming and Flying (to appear).

[3] H.C. Brinkman, "A calculation of the viscous force exerted by a flowing fluid on a dense swarm of particles", App Z. Sci. Res. A1 $(1949), 27-34$.

[4] Chuen-Yen Chow and Ying-Chung Lai, "Alternating flow in trachea", Resp. Physiol. 16 (1972), 22-32.

[5] I.R. Cowan, "Mass, heat and momentum exchange between stands of plants and their atmospheric environment", Quart. J. Roy. Met. Soc. 94 (1968), 523-544.

[6] A. Erdélyi, W. Magnus, F. Oberhettinger, F.G. Tricomi (edited by), Higher transcendental functions, Volume II (based, in part, on notes left by Harry Bateman. McGraw-Hill, New York, Toronto, London, 1953).

[7] John Happel, Howard Brenner, Lower Reynolds number hydro dynamics with special applications to particulate media (Prentice-Hall, Englewood Cliffs, N.J., 1965).

[8] E. Inoue, "On the turbulent structure of airflow within crop canopies", J. Met. Soc. Japan 41 (1963), 317-325.

[9] A.S. Jones, "Wall shear in pulsatile flow", Bulz. Math. Biophys. 34 (1972), 79-86.

[10] George M. Murphy, Ordinary differential equations and their solutions (Van Nostrand Reinhold, London, New York, Cincinati, Toronto, Melbourne, 1960).

[11] P.G. Saffman, "On the boundary conditions at the surface of a porous medium", Studies in App 2. Math. 50 (1951), 93-101.

[12] J.R. Womersley, "Method for the calculation of velocity, rate of flow and viscous drag in arteries when the pressure gradient is known", J. Physiol. 127 (1955), 553-563.

CSIRO,

Division of Mathematics and Statistics,

Canberra, ACT. 